\title{
Critical Review
}

\section{Proteasome Activation as a Novel Antiaging Strategy}

\author{
Niki Chondrogianni and Efstathios S. Gonos \\ National Hellenic Research Foundation, Institute of Biological Research and Biotechnology, Athens 11635, Greece
}

\begin{abstract}
Summary
Homeostasis is a key feature of cellular lifespan. Maintenance of cellular homeostasis influences the rate of aging and is determined by several factors, including efficient proteolysis of damaged proteins. Protein degradation is predominately catalyzed by the proteasome. Specifically, the proteasome is responsible for cell clearance of abnormal, denatured or in general damaged proteins as well as for the regulated degradation of short-lived proteins. As proteasome has an impaired function during aging, emphasis has been given recently in identifying ways of its activation. A number of studies have shown that the proteasome can be activated by genetic manipulations as well as by factors that affect its conformation and stability. Importantly the developed proteasome activated cell lines exhibit an extended lifespan. This review article discusses in details the various factors that are involved in proteasome biosynthesis and assembly and how they contribute to its activation. Finally as few natural compounds have been identified having proteasome activation properties, we discuss the advantages of this novel antiaging strategy. (c) 2008 IUBMB

IUBMB Life, 60(10): 651-655, 2008
\end{abstract}

Keywords aging; homeostasis; longevity; proteasome; protein degradation; senescence.

\section{PROTEASOME, A KEY COMPONENT FOR PROTEIN DEGRADATION AND MAINTENANCE OF CELLULAR HOMEOSTASIS}

Homeostasis is a key feature that determines organismal lifespan. Maintenance of homeostasis is influenced by several intracellular functions, which, in turn, determine the quality and the extent of cellular and organismal lifespan (1). Efficient protein degradation is one of the major factors that contribute to the retention of cellular homeostatic balance.

Received 28 March 2008; accepted 9 April 2008

Address correspondence to: Efstathios S. Gonos, National Hellenic Research Foundation, Institute of Biological Research and Biotechnology, 48 Vas. Constantinou Ave., Athens 11635, Greece. Tel: +30-2107273756. Fax: +30-210-7273677. E-mail: sgonos@eie.gr
Protein degradation is predominately catalyzed by the proteasome. The proteasome is responsible for cell clearance of abnormal, denatured or in general damaged proteins as well as, for the regulated degradation of short-lived proteins $(2,3)$. The $20 \mathrm{~S}$ proteasome, a $700 \mathrm{kDa}$ multisubunit enzyme complex, is a barrel-shape stack of four heptameric rings localized in both cytoplasm and nucleus. The two outer $\alpha$-subunits rings $\left(\alpha_{1-7}\right)$ embrace two central head-to-head oriented rings containing $\beta$-subunits $\left(\beta_{1-7}\right)$. The internal chamber that is composed by $\beta$-subunits hosts the proteolytic active sites. Three of the $\beta$-subunits, $\beta_{1}, \beta_{2}$, and $\beta_{5}$, are responsible for the proteasome hydrolyzing activities that cleave peptide bonds on the carboxyl site of acidic (peptidylglutamylpeptide hydrolyzing activity, PGPH), basic (trypsin-like activity, T-L), and hydrophobic (chymotrypsin-like activity, CT-L) amino acids, respectively (3).

Capping of each side of $20 \mathrm{~S}$ particle by $19 \mathrm{~S}$ regulatory complexes gives rise to $26 \mathrm{~S}$ proteasome that is responsible for the ATP/ubiquitin-dependent protein degradation. The 19S particle is composed of two subcomplexes, namely the lid and the base. The lid covers the base and it is involved in the recognition and ubiquitin chain processing of substrates before their translocation and degradation. The base is consisted of six ATPase subunits involved in the unfolding and further translocation of the substrate (4). The target protein is first labeled via covalent attachment of multiple ubiquitin molecules before the degradation by the $26 \mathrm{~S}$ complex. Three steps are involved in the conjugation of ubiquitin to the substrate. Ubiquitin is activated by $\mathrm{E} 1$, the ubiquitin-activating enzyme, and then transferred by an E2 enzyme, the ubiquitin-carrier protein or ubiquitin-conjugating enzyme, to a member of the ubiquitin-protein ligase family, E3, to which the target protein is specifically bound. This enzyme catalyzes the repeated conjugation of ubiquitin to the substrate prior of the $26 \mathrm{~S}$ complex-mediated degradation (2).

Proteasome has also been implicated in antigen presentation process and immune response, mainly through immunoproteasomes. In this proteasome type, the constitutively expressed $\beta_{1}$, $\beta_{2}$, and $\beta_{5}$ subunits are substituted during de novo proteasome biosynthesis by $\beta_{1 \mathrm{i}}, \beta_{2 \mathrm{i}}$, and $\beta_{5 \mathrm{i}}$ subunits respectively. As a result, although these particles digest proteins at rates similar to 
those for constitutive proteasomes, they generate a higher fraction of peptides with the appropriate C-termini and length to serve in antigen presentation. In immunoproteasomes, PA28/ $11 \mathrm{~S}$ activator can replace the $19 \mathrm{~S}$ complex (5). Finally additional tissues may also have their own type of proteasomes as it was recently shown in cortical thymic epithelial cells (6).

Proteasome biogenesis involves the following accurately ordered multistep events: (a) The biosynthesis of $\alpha$ - and $\beta$ - subunits, (b) The organization of $\alpha$-subunits in seven-member rings under the supervision and assistance of proteasome assembling chaperones, 1, 2, and 3 (PAC1-3), (c) The association of precursor $\beta$-subunits with UMP1/POMP accessory protein that in conjunction to the $\alpha$-subunits rings gives rise to half proteasomes, $(d)$ The dimerization of half proteasomes, and $(e)$ The production of mature $20 \mathrm{~S}$ proteasomes $(7,8)$. As these steps of assembly and maturation are highly organized, they underlie the complexity of proteasome regulation.

\section{PROTEASOME HAS AN IMPAIRED FUNCTION DURING AGING}

Aging is a natural biological process that involves the gradual decline of a variety of physiological functions and the eventual failure of homeostasis. Mammalian aging can be studied in vitro as mitotic cells loose their proliferative capacity following serial passaging, a process termed as "replicative senescence" (9). Senescent cells are characterized by several morphological and biochemical alterations when compared with their young counterparts, including changes in proteasome function. Specifically, a number of studies have shown an $\sim 50 \%$ decline of proteasomal activities in several aged tissues in humans (muscle, lens, lymphocytes, and epidermis) as well as in other mammals such as mice, rats, and bovines (liver, spinal cord, lens, heart, and retina; 10). A similar decline of proteasome activities has also been revealed in human primary cultures undergoing replicative senescence (10), whereas proteasome inhibition in young cells induces premature senescence (11). Earlier work has attributed the observed decrease of proteasome function to the accumulation of damaged proteins, such as lipofuscin, during aging and senescence (12). To understand the molecular basis of these findings, work in our laboratory has determined that the decreased function of proteasome in senescence is, in addition to the accumulation of damaged proteins, primarily due to the reduced rates of proteasome biosynthesis and assembly. Moreover, we have determined that replicative senescence is accompanied with reduced levels of the $\beta$-type subunits, thus acting as the "rate-limiting" subunits for efficient proteasome assembly (13).

Immunoproteasomes have also been investigated during aging. Ponnappan and coworkers (14) have recently reported lower expression of $\alpha$ - and $\beta$ - type proteasome subunits in Tcells derived from old donors, while we have shown inability of senescent human fibroblasts to induce proteasome immunosubunits following interferon- $\gamma$ treatment (15). Interestingly, in tissues irrelevant to the immune system, like the muscle, an age-related upregulation of immunoproteasomes in parallel to the decreased amount of constitutive proteasomes has been demonstrated, thereby implying for a potential compensatory mechanism which may result in an overall proteasome function fine-tuning (16).

Finally, work in 19S complex has identified several subunits to be downregulated during replicative senescence $(13,17)$, whereas a lower ability of proteasome activators to assemble with $20 \mathrm{~S}$ complex in aged rat muscle has also been reported $(16,18)$. In support, recent studies in Drosophila melanogaster have shown that aging also perturbs $26 \mathrm{~S}$ proteasome assembly (19). In summary, these data strongly suggest that aging has profound effects on proteasome biosynthesis, assembly, and function.

\section{MEANS OF PROTEASOME ACTIVATION}

Proteasome can be activated by several regulators, such as the $19 \mathrm{~S}$ and $11 \mathrm{~S}$ complexes, for performing its ordinary functions as described previously. However, over the last years it became evident that proteasome, in addition to its known regulators, can also be activated by genetic or other means.

\section{Genetic Activation of the Proteasome}

Goldberg and coworkers (20) were the first to demonstrate the enhancement of CT-L and T-L proteasome activities following $\beta_{5 \mathrm{i}}$ transfection in lymphoblasts and HeLa cells. The same group in a follow up study has shown a similar stimulation of T-L and PGPH activities following $\beta_{1 \mathrm{i}}$ and $\beta_{1}$ subunit overexpression in HeLa cells (21). More recently, we overexpressed the $\beta_{5}$ subunit stably in WI38/T and HL60 cells (22). We found that transfection of the $\beta_{5}$ subunit resulted to upregulation of other $\beta$-type subunits, thus implying for a common regulatory loop, and to the recruitment of "free" $\alpha$-type subunits to produce new and functional proteasomes. This was evident as the developed "proteasome activated cell lines" exhibited increased rates of proteolysis as well as enhanced resistance following cell treatment with various oxidants. Importantly, a similar $\beta_{5}$ subunit overexpression in primary human embryonic fibroblasts (IMR90 cells) resulted in the extension of lifespan by $\sim 15$ $20 \%$ (22). The observed coregulation of $\beta$-type subunits has also been confirmed in other cell types, such as the lens epithelial cells (23) or the murine neuroblastoma cells (24), following overexpression of the $\beta_{5}$ subunit. Moreover, it was recently shown that restoration of the normal level of proteasome subunits in aged human fibroblasts reduces the levels of various aging biomarkers (25), thereby confirming our assumption regarding the vital association between optimal proteasome function and retention of cellular homeostasis. Finally, we have recently achieved proteasomal upregulation via overexpression of hUMP1/POMP protein (26). UMP/POMP stable clones exhibited increased rates of proteasome assembly and function as assayed by their enhanced recovery ability following administration of oxidative stressors. 


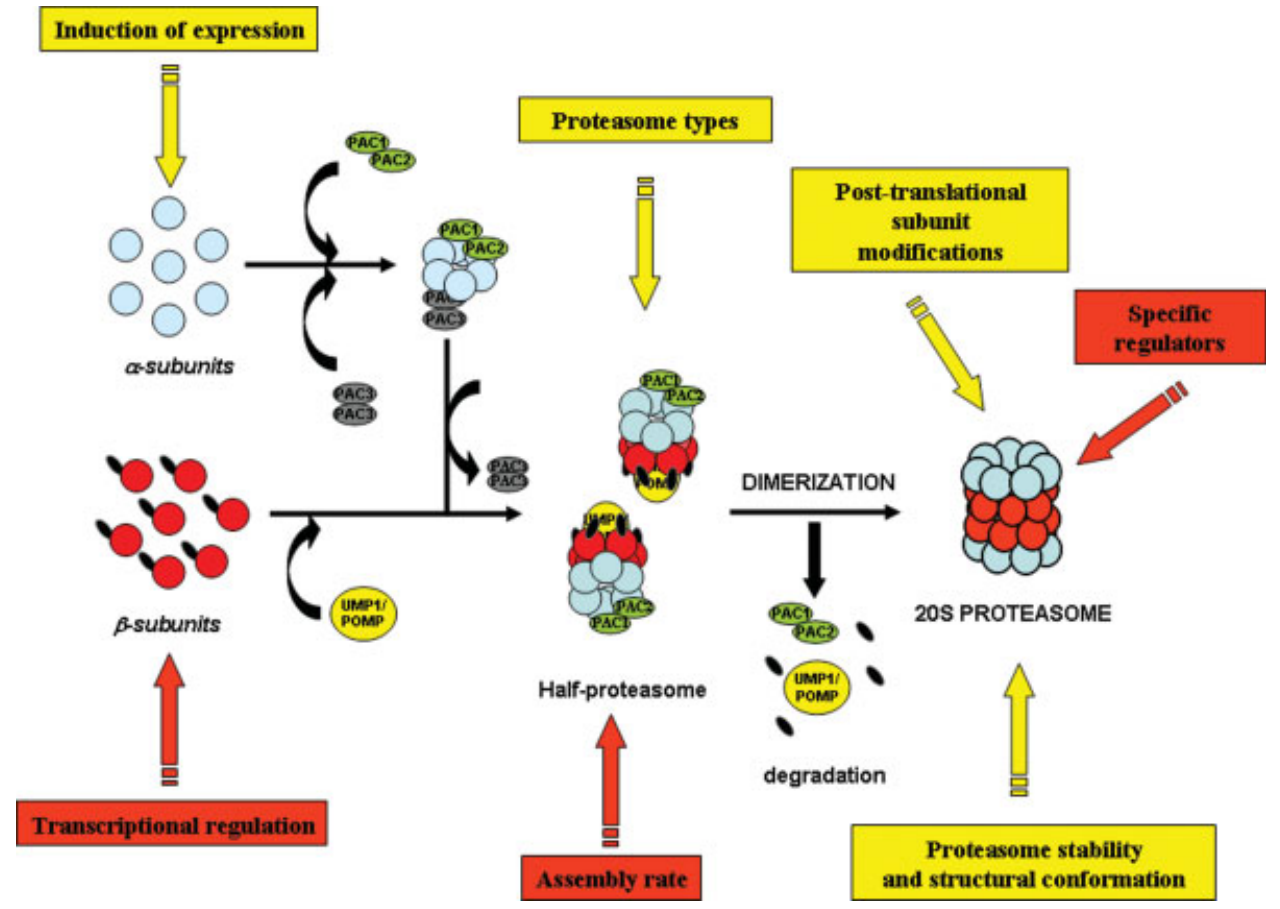

Figure 1. Potential steps of intervention toward proteasome activation. The Figure summarizes the various known steps of proteasome biosynthesis and assembly. Manipulations of factors shown in yellow are known to activate the proteasome (see text for details). Red boxes refer to entirely unexplored pathways of proteasome activation.

\section{Proteasome Activation by Natural or Chemical Compounds}

Effects on Proteasome Conformation. Structural alterations of proteasome subunits have been shown to affect proteasome activity through changes of the $20 \mathrm{~S}$ barrel conformation. Initially, SDS and some fatty acids have been shown to stimulate proteasome activities in the test tube (27), whereas potassium chloride has a negative effect (28) by favoring the open or the closed conformation of the proteasome, respectively. Proteasome-activating hydrophobic peptides have been shown to be bound as modifiers at noncatalytic sites, thus mimicking the effect of $11 \mathrm{~S}$ complex by opening the $\alpha$-gated pore (29). On the basis of these studies, we have recently isolated oleuropein, the most abundant of the phenolic compounds in Olea europaea leaf extract, olive oil, and olives (30), and we have demonstrated that it has a stimulatory impact on proteasome activities in vitro. The increased activities promote cellular resistance to oxidants and confer extension of human fibroblasts lifespan (31). Oleuropein most likely acts through structural changes of the 20S $\alpha$-gated channels conformation in a similar manner to SDS, albeit its effects are considerably stronger. A similar stimulatory effect has been observed by an algae extract on human keratinocytes, as it provides protection from UVA and UVB irradiation (32).

Effects on Expression of Proteasome Subunits. Proteasome activation has also been achieved following treatment with vari- ous antioxidants. Specifically, Kensler and coworkers have shown natural antioxidants, such as dithiolethione and sulforafane, to enhance mammalian proteasome expression through the Keap1-Nrf2 signaling pathway, resulting to increased protection against various oxidants $(24,33)$. Nevertheless, the correlation between aging and Nrf2 pathway is not established, since Nrf2 knock out mice were not identified to exhibit further alterations of lifespan following caloric restriction (34).

\section{In Vivo Evidence of Proteasome Activation}

The previously reported studies establish the beneficial effects of proteasome activation in various in vitro systems. To test the in vivo relevance of these findings, we have determined proteasome biosynthesis and function in cultures derived from skin fibroblasts biopsies of several healthy donors of various ages including healthy centenarians. Although we observed a proteasome function decline with the age of the donors, importantly, we found that healthy centenarians exhibit a more functional proteasome when compared with the elderly (35). We hypothesize that their functional proteasome, by providing enhanced rates of proteolysis of damaged proteins, indirectly contributes to their longevity. In support various studies have shown that in caloric restricted animals the proteasome function is maintained or even enhanced, thus possibly contributing to the observed extended lifespan of these animals (36-38). 


\section{CONCLUSIONS AND FUTURE PERSPECTIVES}

This review highlights the different means of proteasome activation and its beneficial effects in the maintenance of cellular homeostasis. Given these intriguing findings, a number of arising questions regarding the identification of the molecular pathways that result to proteasome biosynthesis and function and their effects in several biological phenomena, such as aging, remain to be addressed.

First, the transcription factor(s) that is (are) responsible for mammalian proteasomes expression are essentially not known, apart from scattered reports which have implicated NF-kB (39) and Nrf2 (33) (Fig. 1; Induction of expression). An equally important question is to understand how the overexpression of a single $\beta$-subunit result in the coregulated expression of other $\beta$ type subunits (Fig. 1; Transcriptional regulation). Moreover, future work should unravel the mechanism of recruitment of free $\alpha$-type subunits (following the overexpression of $\beta$-type subunits) as well as the role of factors that contribute to proteasome assembly, such as the newly identified chaperones PAC13 (8) and UMP1/POMP (40) (Fig. 1; Assembly rates). Similarly, the forces/signals that govern the assembly of half proteasomes and their dimerization to produce active complexes (Fig. 1; Assembly rates) or the compensatory mechanisms that induce immunoproteasomes expression in replacement of the reduced constitutive complexes are still unexplored (Fig. 1; Proteasome types). A novel protein that interacts with proteasomal ATPases, proteasomal ATPase-associated factor 1 (PAAF1), was isolated and shown to act as negative regulator of the proteasome activities by affecting the assembly/disassembly of the $26 \mathrm{~S}$ complex (41). Thus it would not be surprising if future work identifies a similar factor with positive effect on $26 \mathrm{~S}$ stability or regulators of the ATPases activities (Fig. 1; Specific regulators). Some posttranslational modifications of $20 \mathrm{~S}$ or $19 \mathrm{~S}$ complexes subunits that affect proteasome activities and the 20S:19S stability have been identified $(42,43)$, but only few of them have been investigated during aging (44) (Fig. 1; Posttranslational modifications). Finally, the identification of natural compounds that induce conformational (Fig. 1; Proteasome stability and structural conformation) or in general qualitative alterations in proteasome complexes and that would affect its activities and functions constitute a strong bet in both the proteasome and the aging fields (Fig. 1; Induction of expression). Figure 1 recapitulates these possible steps of intervention toward proteasome activation during the assembly process. Despite the increasing number of studies regarding proteasome activation and the accomplished progress, many areas of proteasome regulation are not fully elucidated. Antiaging strategies should aim on the controlled preservation and activation of proteasome function.

\section{ACKNOWLEDGEMENTS}

Cited work from our laboratory was supported by a European Union Integrated Project/FP-6 grant ("Proteomage", LSHMCT-518230) to ESG.

\section{REFERENCES}

1. Petropoulou, C., Chondrogianni, N., Simões, D., Agiostratidou, G., Drosopoulos, N., Kotsota, V., and Gonos, E. S. (2000) Aging and longevity. A paradigm of complementation between homeostatic mechanisms and genetic control? Ann. N.Y. Acad. Sci. 908, 133-142.

2. Ciechanover A. (2005) Intracellular protein degradation: from a vague idea through the lysosome and the ubiquitin-proteasome system and onto human diseases and drug targeting. Cell Death Differ. 12, 11781190 .

3. Goldberg, A. L. (2007) Functions of the proteasome: from protein degradation and immune surveillance to cancer therapy. Biochem. Soc. Trans. 35, 12-17.

4. Hanna, J. and Finley, D. (2007) A proteasome for all occasions. FEBS Lett. 581, 2854-2861.

5. Borissenko, L. and Groll, M. (2007) Diversity of proteasomal missions: fine tuning of the immune response. Biol. Chem. 388, 947-955.

6. Murata, S., Sasaki, K., Kishimoto, T., Niwa, S., Hayashi, H., Takahama, Y., and Tanaka, K. (2007) Regulation of CD8 + T cell development by thymus-specific proteasomes. Science 316, 1349-1353.

7. Heinemeyer, W., Ramos, P. C., and Dohmen, R. J. (2004) The ultimate nanoscale mincer: assembly, structure and active sites of the $20 \mathrm{~S}$ proteasome core. Cell. Mol. Life Sci. 61, 1562-1578.

8. Hirano, Y., Hayashi, H., Iemura, S., Hendil, K. B., Niwa, S., Kishimoto, T., Kasahara, M., Natsume, T., Tanaka, K., and Murata, S. (2006) Cooperation of multiple chaperones required for the assembly of mammalian 20S proteasomes. Mol. Cell 24, 977-984.

9. Herbig, U. and Sedivy, J. M. (2006) Regulation of growth arrest in senescence: Telomere damage is not the end of the story. Mech. Ageing Dev. 127, 16-24.

10. Chondrogianni, N. and Gonos, E. S. (2005) Proteasome dysfunction in mammalian aging: steps and factors involved. Exp. Gerontol. 40, 931938.

11. Chondrogianni, N. and Gonos, E. S. (2004) Proteasome inhibition induces a senescence-like phenotype in primary human fibroblasts cultures. Biogerontology 5, 55-61.

12. Grune, T., Jung, T., Merker, K., and Davies, K. J. (2004) Decreased proteolysis caused by protein aggregates, inclusion bodies, plaques, lipofuscin, ceroid, and 'aggresomes' during oxidative stress, aging, and disease. Int. J. Biochem. Cell Biol. 36, 2519-2530.

13. Chondrogianni, N., Stratford, F. L. L., Trougakos, I. P., Friguet, B., Rivett, A. J., and Gonos, E. S. (2003) Central role of the proteasome in senescence and survival of human fibroblasts: induction of a senescence-like phenotype upon its inhibition and resistance to stress upon its activation. J. Biol. Chem. 278, 28026-28037.

14. Ponnappan, S., Ovaa, H., and Ponnappan, U. (2007) Lower expression of catalytic and structural subunits of the proteasome contributes to decreased proteolysis in peripheral blood $\mathrm{T}$ lymphocytes during aging. Int. J. Biochem. Cell. Biol. 39, 799-809.

15. Stratford, F. L. L., Chondrogianni, N., Trougakos, I. P., Gonos, E. S., and Rivett, A. J. (2006) Proteasome response to interferon-gamma is altered in senescent human fibroblasts. FEBS Lett. 580, 39893994.

16. Ferrington, D. A., Husom, A. D., and Thompson, L. V. (2005) Altered proteasome structure, function and oxidation in aged muscle. FASEB $J$. 19, 644-646.

17. Ly, D. H., Lockhart, D. J., Lerner, R. A., and Schultz, P. G. (2000) Mitotic misregulation and human aging. Science 287, 2486-2492.

18. Husom, A. D., Peters, E. A., Kolling, E. A., Fugere, N. A., Thompson, L. V., and Ferrington, D. A. (2004) Altered proteasome function and subunit composition in aged muscle. Arch. Biochem. Biophys. 421, 6776.

19. Vernace, V. A., Arnaud, L., Schmidt-Glenewinkel, T., and FigueiredoPereira, M. E. (2007) Aging perturbs $26 \mathrm{~S}$ proteasome assembly in Drosophila melanogaster. FASEB J. 21, 2672-2682. 
20. Gaczynska, M., Rock, K., Spies, T., and Goldberg, A. L. (1994) Peptidase activities of proteasomes are differentially regulated by the major histocompatibility complex-encoded genes for LMP2 and LMP7. Proc. Natl. Acad. Sci. USA 91, 9213-9217.

21. Gaczynska, M., Goldberg, A. L., Tanaka, K., Hendil, K. B., and Rock, K. (1996) Proteasome subunits X and Y alter peptidase activities in opposite ways to the interferon-gamma-induced subunits LMP2 and LMP7. J. Biol. Chem. 271, 17275-17280.

22. Chondrogianni, N., Tzavelas, C., Pemberton, A. J., Nezis, I. P., Rivett, A. J., and Gonos, E. S. (2005) Overexpression of proteasome beta 5 subunit increases the amount of assembled proteasome and confers ameliorated response to oxidative stress and higher survival rates. J. Biol. Chem. 280, 11840-11850.

23. Liu, Y., Liu, X., Zhang, T., Luna, C., Liton, P. B., and Gonzalez, P. (2007) Cytoprotective effects of proteasome bet5 subunit overexpression in lens epithelial cells. Mol. Vis. 13, 31-38.

24. Kwak, M. K., Cho, J. M., Huang, B., Shin, S., and Kensler, T. W. (2007) Role of increased expression of the proteasome in the protective effects of sulphoraphane against hydrogen peroxide-mediated cytotoxicity in murine neuroblastoma cells. Free Radic. Biol. Med. 43, 809-817.

25. Hwang, J. S., Hwang, J. S., Chang, I., and Kim, S. (2007) Age-associated decrease in proteasome content and activities in human dermal fibroblasts: restoration of normal level of proteasome subunits reduces aging markers in fibroblasts from elderly persons. J. Gerontol. A Biol. Sci. Med. Sci. 62, 490-499.

26. Chondrogianni, N. and Gonos, E. S. (2007) Overexpression of hUMP1/ POMP proteasome accessory protein enhances proteasome-mediated antioxidant defence. Exp. Gerontol. 42, 899-903.

27. Dahlmann, B., Rutschmann, M., Kuehn, L., and Reinauer, H. (1985) Activation of the multicatalytic proteinase from rat skeletal muscle by fatty acids or sodium dodecyl sulphate. Biochem. J. 228, 171-177.

28. Kohler, A., Cascio, P., Leggett, D. S., Woo, K. M., Goldberg, A. L., and Finley, D. (2001) The axial channel of the proteasome core particle is gated by the Rpt2 ATPase and controls both substrate entry and product release. Mol. Cell 7, 1143-1152.

29. Kisselev, A. F., Kaganovich, D., and Goldberg, A. L. (2002) Binding of hydrophobic peptides to several non-catalytic sites promotes peptide hydrolysis by all active sites of $20 \mathrm{~S}$ proteasomes. Evidence for peptideinduced channel opening in the alpha-rings. J. Biol. Chem. 277, 2226022270.

30. Bonoli, M. and Bendini, A. (2004) Qualitative and semiquantitative analysis of phenoloic compounds in extra virgin olive oils as a function of the ripening degree of olive fruits by different analytical techniques. J. Agric. Food Chem. 52, 7026-7032.

31. Katsiki, M., Chondrogianni, N., Chinou, I., Rivett, A. J., and Gonos, E. S. (2007) The olive constituent oleuropein exhibits proteasome stimulatory properties in vitro and confers life span extension of human embryonic fibroblasts. Rejuvenation Res. 10, 157-172.

32. Bulteau, A. L., Moreau, M., Saunois, A., Nizard, C., and Friguet, B. (2006) Algae extract-mediated stimulation and protection of proteasome activity within human keratinocytes exposed to UVA and UVB irradiation. Antioxid. Redox Signal. 8, 136-143.

33. Kwak, M. K., Wakabayashi, N., Greenlaw, J. L., Yamamoto, M., and Kensler, T. W. (2003) Antioxidants enhance mammalian proteasome expression through the Keap1-Nrf2 signaling pathway. Mol. Cell. Biol. 23, 8786-8794.

34. Pearson, K.J., Lewis, K.N., Price, N.L., Chang, J.W., Perez, E., Cascajo, M. V., Tamashiro, K.L., Poosala, S., Csiszar, A., Ungvari, Z., Kensler, T. W., Yamamoto, M., Egan, J.M., Longo, D. L., Ingram, D.K., Navas, P., and de Cabo, R. (2008) Nrf2 mediates cancer protection but not prolongevity induced by caloric restriction. Proc. Natl. Acad. Sci. 105 , 2325-2330.

35. Chondrogianni, N., Petropoulos, I., Franceschi, C., Friguet, B., and Gonos, E. S. (2000) Fibroblast cultures from healthycentenarians have an active proteasome. Exp. Gerontol. 35, 721-728.

36. Bonelli, M. A., Desenzani, S., Cavallini, G., Donati, A., Romani, A. A., Bergamini, E., and Borghetti, A. F. (2008) Low-level caloric restriction rescues proteasome activity and Hsc70 level in liver ofaged rats. Biogerontology 9, 1-10.

37. Goto, S., Takahashi, R., Radak, Z., and Sharma, R. (2007) Beneficial biochemical outcomes of late-onset dietary restriction in rodents. Ann. N.Y. Acad. Sci. 1100, 431-441.

38. Salesby, J. T., Judge, A. R., Yimlamai, T., Leeuwenburgh, C., and Dodd, S. L. (2005) Life long calorie restriction increases heat shock proteins and proteasome activity in soleus muscles of Fisher 344 rats. Exp. Gerontol. 40, 37-42.

39. Du, J., Mitch, W. E., Wang, X., and Price, S. R. (2000) Glucocorticoids induce proteasome $\mathrm{C} 3$ subunit expression in L6 muscle cells by opposing the suppression of its transcription by NF-kappa B. J. Biol. Chem. 275, 19661-19666.

40. Burri, L., Hockendorff, J., Boehm, U., Klamp, T., Dohmen, R. J., and Lévy, F. (2000) Identification and characterization of a mammalian protein interacting with $20 \mathrm{~S}$ proteasome precursors. Proc. Natl. Acad. Sci. USA 97, 10348-10353.

41. Park, Y., Hwang, Y.-P., Lee, J.-S., Seo, S.-H., Yoon, S. K., and Yoon, Y.-B. (2005) Proteasomal ATPase-associated facto 1 negatively regulates proteasome activity by interacting with proteasomal ATPases. Mol. Cell. Biol. 25, 3842-3853.

42. Rivett, A. J., Bose, S., Brooks, P., and Broadfoot, K. I. (2001) Regulation of proteasome complexes by $\gamma$-interferon and phosphorylation. Biochimie 83, 363-366.

43. Bose, S., Stratford, F. L. L., Broadfoot, K. I., Mason, G. G. F., and Rivett, A. J. (2004) Phosphorylation of 20S proteasome alpha subunit C8 $\left(\alpha_{7}\right)$ stabilizes the $26 \mathrm{~S}$ proteasome and plays a role in the regulation of proteasome complexes by $\gamma$-interferon. Biochem. J. 378, 177-184.

44. Carrard, G., Dieu, M., Raes, M., Toussaint, O., and Friguet, B. (2003) Impact of aging on proteasome structure and function in human lymphocytes. Int. J. Biochem. Cell Biol. 35, 728-739. 